\title{
Discriminatory interaction behaviour of lipid vesicles towards diversely emissive carbon dots synthesized from ortho, meta and para isomeric carbon precursors
}

Nishu Kanwa, Kavana M and Anjan Chakraborty*

Discipline of Chemistry

Indian Institute of Technology Indore, Indore, Madhya Pradesh, India, 453552.

Authors for correspondence

Email: anjanc@iiti.ac.in 


\section{Supporting Information}

Figure S1: Fluorescence emission spectra of C-dots at different excitation wavelengths as indicated, showing excitation dependent emission of a) oCD; b) mCD and c) pCD.
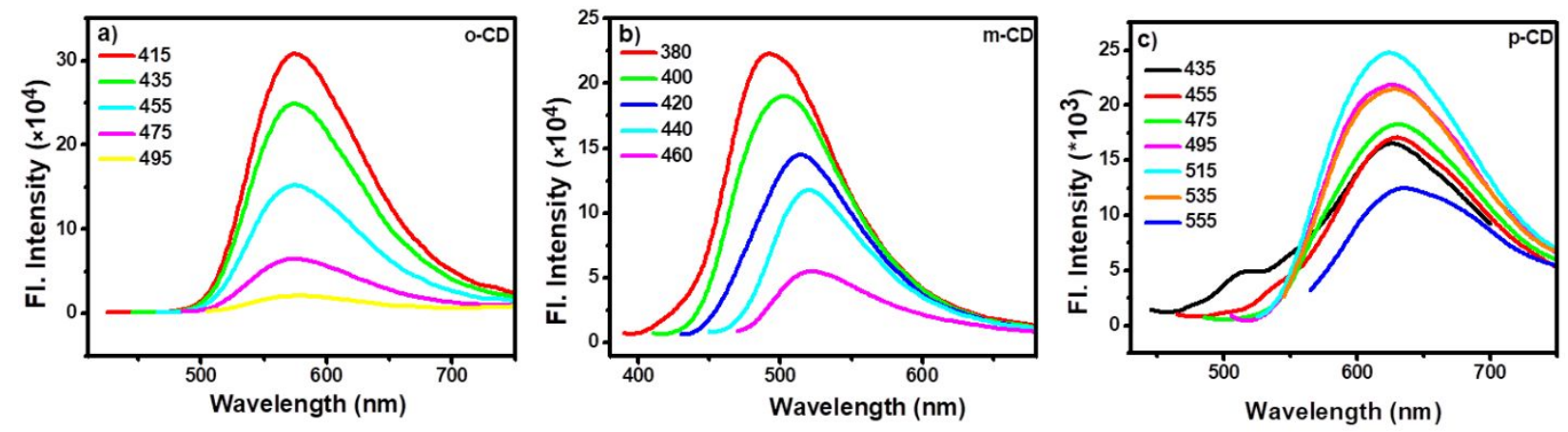

Figure S2: AFM images of synthesized C-dots for a) $\mathrm{oCD}, \mathrm{b}) \mathrm{mCD}$ and c) pCD; and heightprofile analysis along the corresponding lines (bottom).

a)

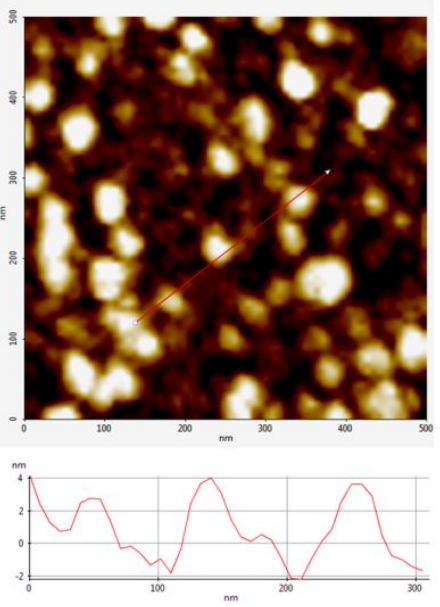

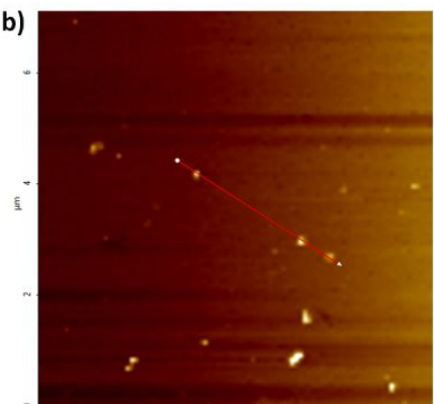
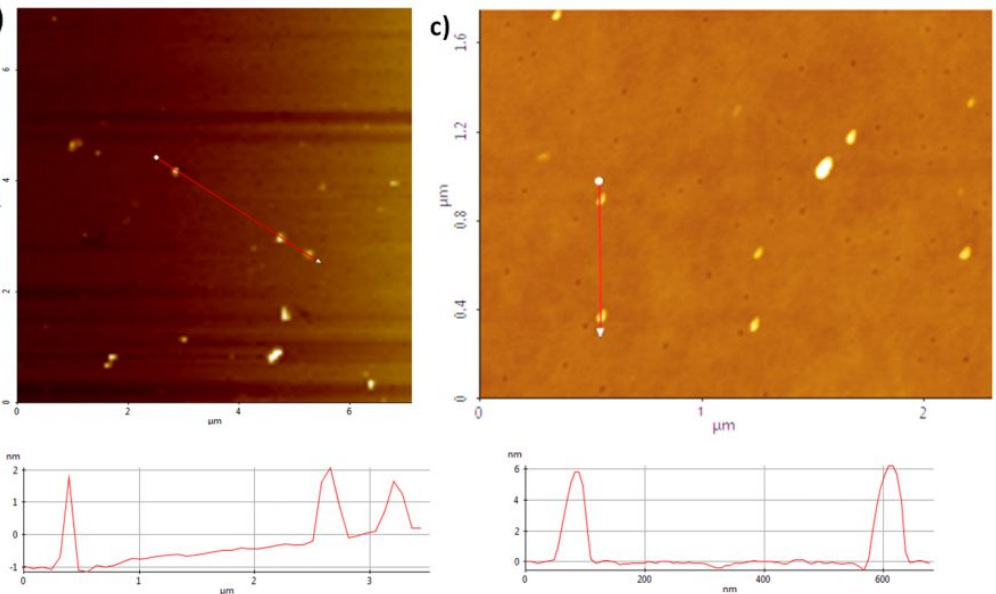

Figure S3: FTIR spectra of the synthesized C-dots for a) oCD; b) mCD and c) pCD.
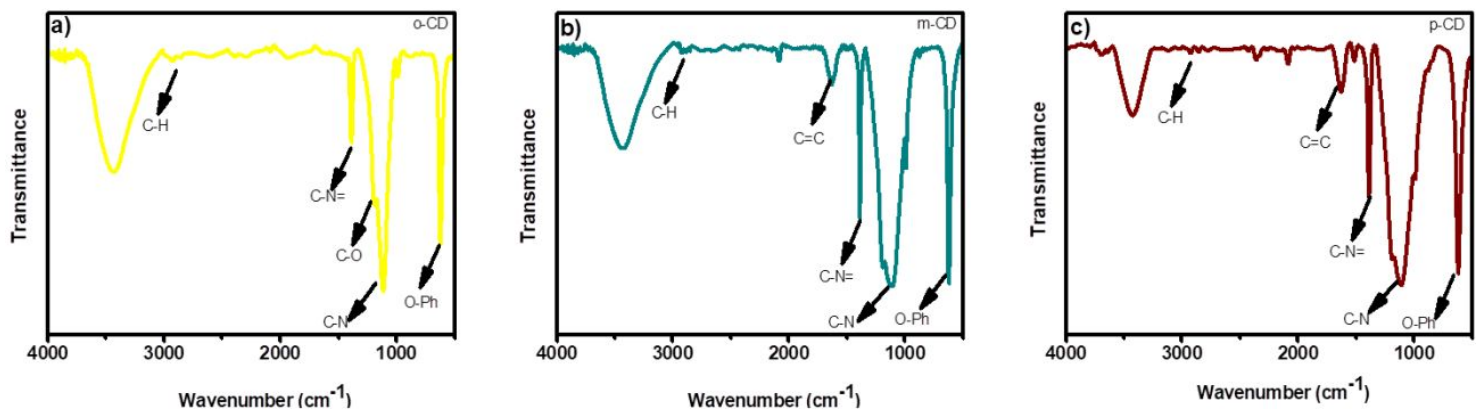
Figure S4: Spectral overlap of the normalized absorbance (red) of the C-dots and normalized fluorescence emission spectra (blue) of the DMPC-embedded PRODAN in a) oCD; b) b) mCD and c) pCD.
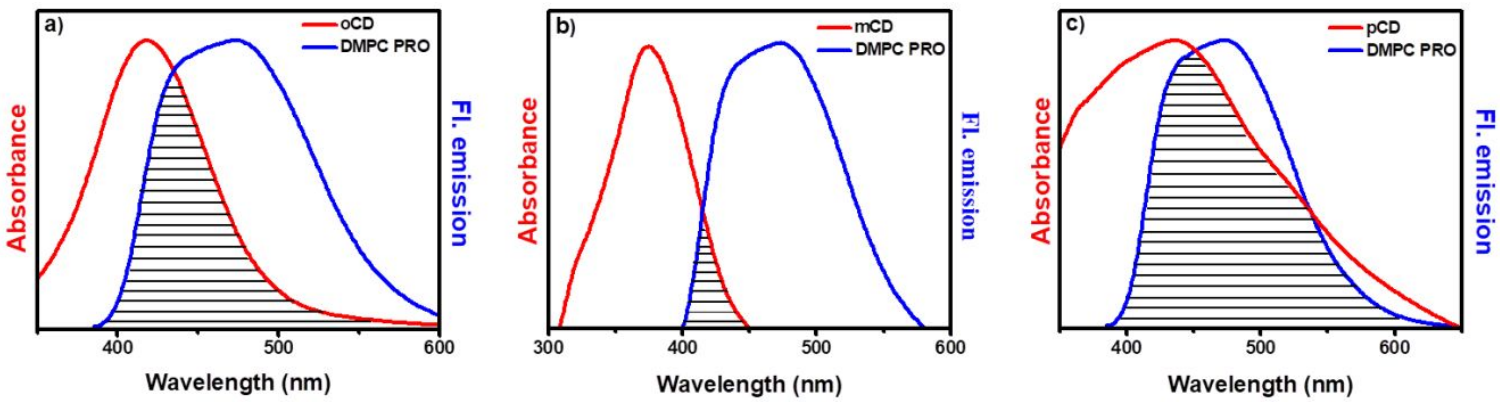

Figure S5: Control experiments for the interaction of blank PRODAN with C-dots for a) oCD, b) $\mathrm{mCD}$ and c) pCD. Here, concentration of PRODAN is fixed at $2 \mu \mathrm{M}$ while varying the concentration of $\mathrm{C}$-dots from 0 to $30 \mu \mathrm{g} / \mathrm{mL}$.
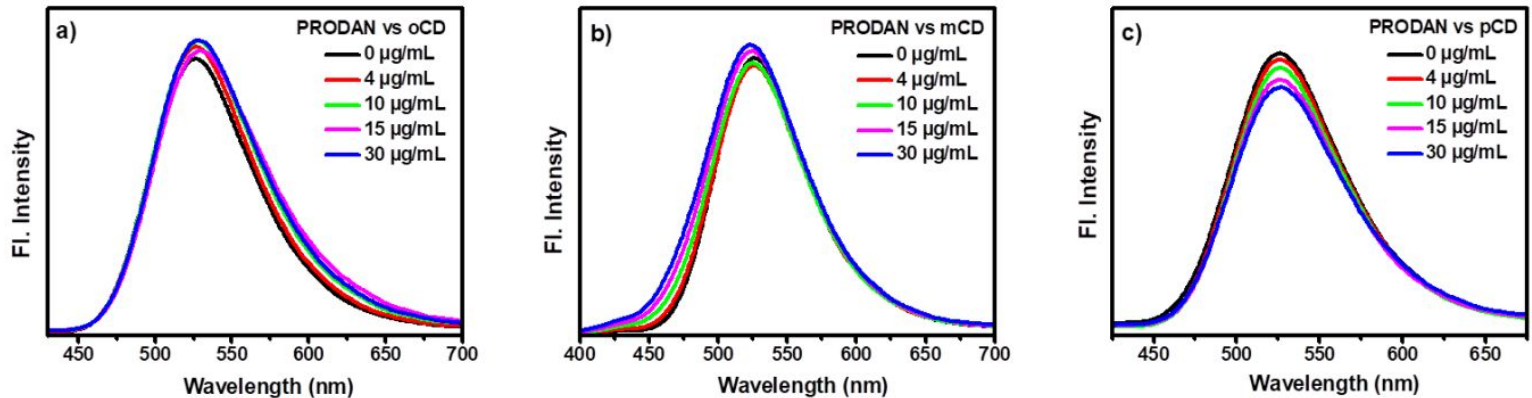

Figure S6: Control experiments for the increasing concentrations of C-dots dispersed in Milli-Q water for a) oCD, b) mCD and c) pCD.
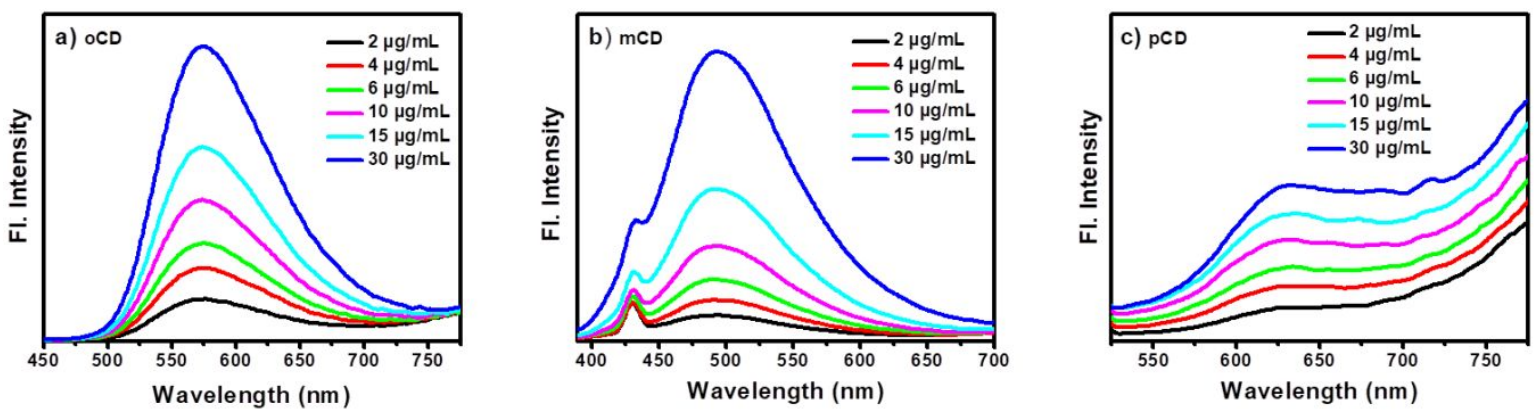

Figure S7: Fluorescence emission spectra of the DPPC-PRODAN upon addition of acceptor C-dots for a) oCD; b) mCD and c) pCD; and corresponding lifetime decay curves (d-f). 

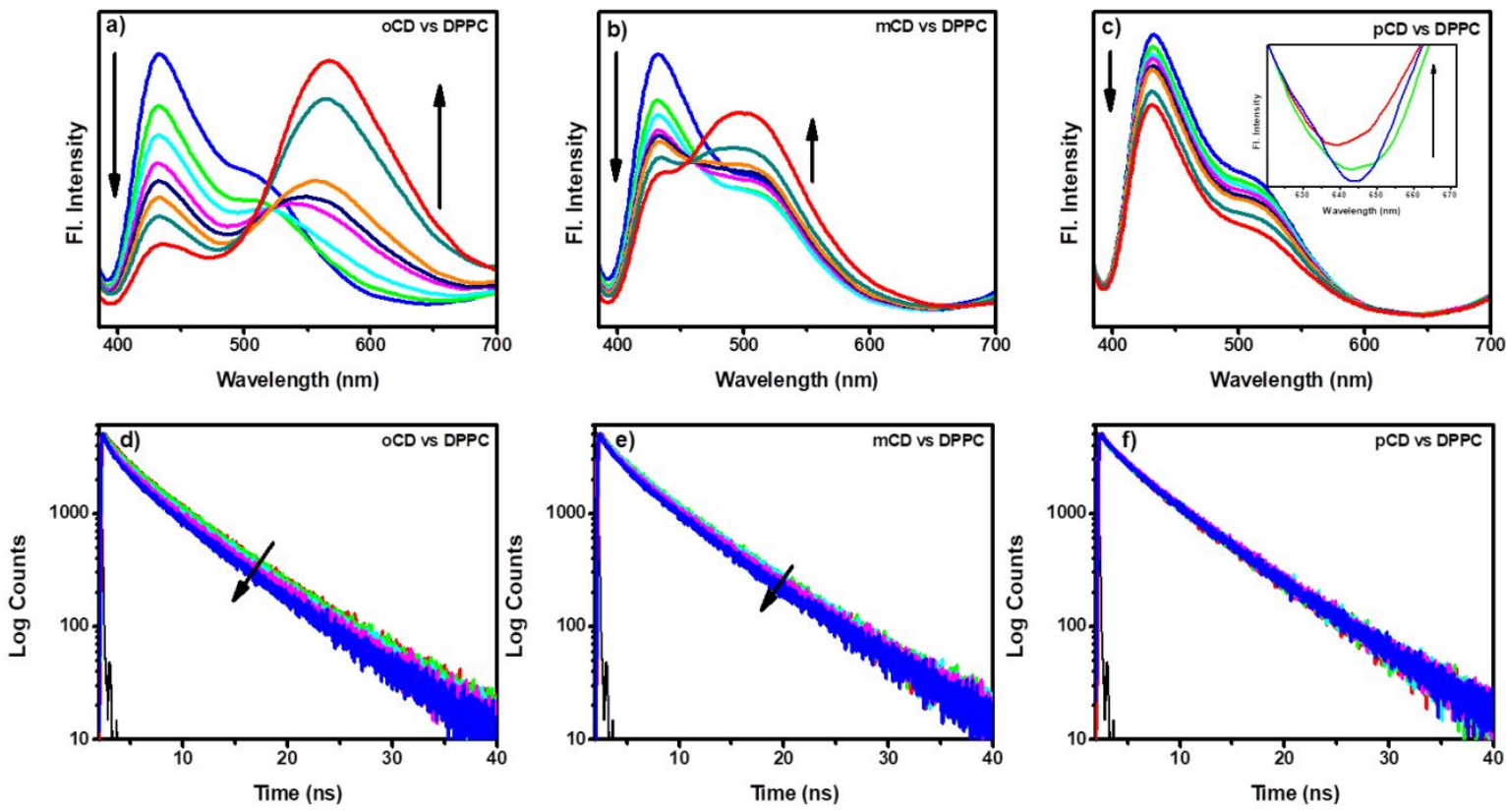

Figure S8: Confocal (emission range 410-480 $\mathrm{nm}$ for a) and 510-610 for (b); bright field (c) and merged images (d) DMPC-pCD system.
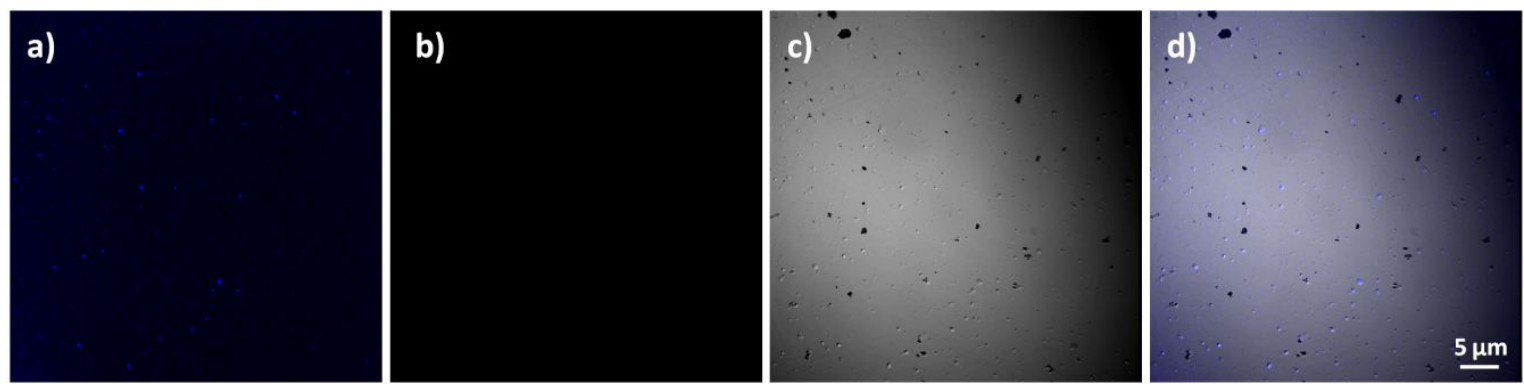

Table S1: Time-resolved decay parameters of C-dots. Lifetime components, normalized amplitudes of lifetime components and average lifetime of $\mathrm{C}$-dots recorded at $\mathrm{T}=25^{\circ} \mathrm{C}$.

\begin{tabular}{|l|c|c|c|c|c|c|c|c|}
\hline Sample & $\tau_{1}(\mathrm{~ns})$ & $\tau_{2}(\mathrm{~ns})$ & $\tau_{3}(\mathrm{~ns})$ & $a_{1}$ & $a_{2}$ & $a_{3}$ & $<\tau_{\text {avg }}>(\mathrm{ns})$ & $\chi^{2}$ \\
\hline \multicolumn{7}{l}{} \\
\hline
\end{tabular}




\begin{tabular}{|l|l|l|l|l|l|l|l|l|}
\hline pCD & 0.67 & 1.38 & 0.10 & 0.39 & 0.18 & 0.43 & 0.55 & 1.04 \\
\hline
\end{tabular}

Table S2: Time-resolved decay parameters of C-dots in presence of DMPC lipid vesicles. Lifetime components, normalized amplitudes of lifetime components and average lifetime of C-dots recorded at $\mathrm{T}=25^{\circ} \mathrm{C}$.

\begin{tabular}{|c|c|c|c|c|c|c|c|c|}
\hline Sample & $\tau_{1}(\mathrm{~ns}$ & $\tau_{2}(\mathbf{n s})$ & $\tau_{3}(\mathrm{~ns})$ & $\mathbf{a}_{1}$ & $\mathbf{a}_{2}$ & $\mathbf{a}_{3}$ & $<\tau_{\text {avg }}>(n s)$ & $\chi^{2}$ \\
\hline & \multicolumn{8}{|c|}{ Laser used: $405 \mathrm{~nm}$, Collected at: $572 \mathrm{~nm}, \mathrm{~T}=25^{\circ} \mathrm{C}$} \\
\hline $\mathbf{O C D}$ & 1.63 & - & - & 1.00 & - & - & 1.63 & 1.13 \\
\hline \multirow{2}{*}{$\begin{array}{l}\text { oCD } \\
\text { DMPC }\end{array}$} & 1.50 & 3.08 & - & 0.79 & 0.21 & - & 1.82 & 1.16 \\
\hline & \multicolumn{8}{|c|}{ Laser used: $375 \mathrm{~nm}$, Collected at: $500 \mathrm{~nm}, \mathrm{~T}=\mathbf{2 5 ^ { \circ }} \mathrm{C}$} \\
\hline $\mathbf{m C D}$ & 1.23 & 7.56 & - & 0.36 & 0.64 & - & 5.28 & 1.21 \\
\hline \multirow{2}{*}{$\begin{array}{l}\text { mCD } \\
\text { DMPC }\end{array}$} & 1.08 & 7.53 & - & 0.32 & 0.68 & - & 5.45 & 1.21 \\
\hline & \multicolumn{8}{|c|}{ Laser used: $445 \mathrm{~nm}$, Collected at: $635 \mathrm{~nm}, \mathrm{~T}=25^{\circ} \mathrm{C}$} \\
\hline pCD & 0.52 & 1.66 & 0.03 & 0.04 & 0.01 & 0.95 & 0.07 & 1.13 \\
\hline $\begin{array}{l}\text { pCD } \\
\text { DMPC }\end{array}$ & 0.38 & 2.00 & 0.009 & 0.00 & 0.00 & 1.00 & 0.01 & 1.10 \\
\hline
\end{tabular}

Table S3: Fluorescence emission quenching percentage and energy transfer efficiency as calculated from time-resolved lifetime for the addition of C-dots to DMPC-PRODAN solution. Stern-Volmer constants $\left(K_{\text {sv }}\right)$ as obtained from the fluorescence emission $\left(F_{0} / F\right)$ vs concentration $(C)$ plot and time-resolved lifetime decay $\left(\tau_{0} / \tau\right)$ vs concentration plot.

\begin{tabular}{|c|c|c|c|c|}
\hline Acceptor C-dot & $\begin{array}{c}\text { Fluorescence } \\
\text { quenching \% } \\
\text { (steady state) }\end{array}$ & $\begin{array}{c}\text { Energy transfer } \\
\text { efficiency (from time } \\
\text { resolved decay) }\end{array}$ & $\begin{array}{c}\mathbf{F}_{\mathbf{0}} / \text { F vs C } \\
\text { slope (steady } \\
\text { state) }\end{array}$ & $\begin{array}{c}\tau_{0} / \tau \text { vs C } \\
\text { slope (time- } \\
\text { resolved) }\end{array}$ \\
\hline oCD & $69 \%$ & $66 \%$ & 0.0817 & 0.0650 \\
\hline mCD & $56 \%$ & $40 \%$ & 0.0443 & 0.0222 \\
\hline pCD & $41 \%$ & $15 \%$ & $\mathbf{0 . 0 2 1 4}$ & $\mathbf{0 . 0 0 6 4}$ \\
\hline
\end{tabular}


Table S4: Time-resolved decay parameters of DMPC-PRODAN in presence of C-dots. Lifetime components, normalized amplitudes of lifetime components and average lifetime recorded at $\mathrm{T}=25^{\circ} \mathrm{C}$.

\begin{tabular}{|l|c|c|c|c|c|c|c|c|}
\hline $\begin{array}{l}\text { Sample } \\
(\mu \mathrm{g} / \mathrm{mL})\end{array}$ & $\tau_{1}(\mathrm{~ns})$ & $\tau_{2}(\mathrm{~ns})$ & $\tau_{3}(\mathrm{~ns})$ & $a_{1}$ & $a_{2}$ & $a_{3}$ & $\begin{array}{c}<\tau_{\text {avg }} \\
(\mathrm{ns})\end{array}$ & $\chi^{2}$ \\
\hline $\begin{array}{l}\text { DMPC } \\
\text { PRODAN }\end{array}$ & 1.70 & 4.31 & - & 0.41 & 0.59 & - & 3.25 & 1.15 \\
\hline oCD 4 & 1.38 & 3.81 & - & 0.50 & 0.50 & - & 2.60 & 1.08 \\
\hline oCD 10 & 1.09 & 3.41 & - & 0.59 & 0.41 & - & 2.05 & 1.22 \\
\hline oCD 15 & 1.64 & 0.55 & 3.74 & 0.44 & 0.36 & 0.20 & 1.68 & 1.20 \\
\hline oCD 20 & 1.42 & 0.43 & 3.48 & 0.46 & 0.37 & 0.17 & 1.40 & 1.15 \\
\hline oCD 30 & 1.22 & 0.33 & 3.25 & 0.46 & 0.42 & 0.12 & 1.09 & 1.25 \\
\hline mCD 4 & 1.52 & 4.09 & - & 0.48 & 0.52 & - & 2.81 & 1.19 \\
\hline mCD 10 & 1.39 & 3.95 & - & 0.54 & 0.46 & - & 2.56 & 1.13 \\
\hline mCD 15 & 1.28 & 3.89 & - & 0.57 & 0.43 & - & 2.41 & 1.15 \\
\hline mCD 20 & 1.25 & 3.94 & - & 0.58 & 0.42 & - & 2.37 & 1.11 \\
\hline mCD 30 & 1.90 & 4.71 & 0.59 & 0.47 & 0.18 & 0.35 & 1.93 & 1.22 \\
\hline pCD 4 & 1.64 & 4.14 & - & 0.43 & 0.57 & - & 3.09 & 1.15 \\
\hline pCD 10 & 1.63 & 4.22 & - & 0.43 & 0.57 & - & 3.05 & 1.11 \\
\hline pCD 15 & 1.58 & 4.05 & - & 0.45 & 0.55 & - & 2.93 & 1.13 \\
\hline pCD 20 & 1.64 & 4.24 & - & 0.45 & 0.55 & - & 3.06 & 1.06 \\
\hline pCD 30 & 1.46 & 3.91 & - & 0.48 & 0.52 & - & 2.74 & 1.13 \\
\hline & & & & & & & & \\
\hline
\end{tabular}

